EGU21-12615

https://doi.org/10.5194/egusphere-egu21-12615

EGU General Assembly 2021

(c) Author(s) 2021. This work is distributed under

the Creative Commons Attribution 4.0 License.

\title{
Agricultural decision-making under uncertainty: a loss function on the kriging variance from soil properties predicted by infrared and X-ray fluorescence spectroscopy
}

Timo Breure ${ }^{1,2}$, Stephan M. Haefele ${ }^{1}$, Richard Webster ${ }^{1}$, Jacqueline A. Hannam², Ronald Corstanje², and Alice E. Milne ${ }^{1}$

${ }^{1}$ Rothamsted Research, SAS, Harpenden, United Kingdom of Great Britain - England, Scotland, Wales

${ }^{2}$ Cranfield University, CEAl, Cranfield, United Kingdom of Great Britain - England, Scotland, Wales

Obtaining detailed soil information at a scale suitable for variable rate application (VRA) of fertilizer requires intense sampling. Within this context, traditional wet chemistry analysis of the soil is too costly to provide sufficient detail on the spatial variation of soils within fields. Infrared and X-ray fluorescence spectroscopy of soil is much cheaper and might provide sufficient data to map the concentrations affordably. Estimates of the concentrations from spectroscopy are subject to error, however, and these errors in turn carry with them costs if they lead to under- or over-applications of fertilizer and resultant loss of potential crop yields or environmental pollution. Given a loss function and an error distribution for the estimates of the concentrations, it is possible to minimize the expected loss from VRA fertilizer management.

Within this study, we have estimated the variation of available $\mathrm{P}$ and $\mathrm{K}$ within horticultural fields and the effect of errors on the expected loss. The topsoil $(0-25 \mathrm{~cm})$ of four fields was sampled at numerous points and analysed by infrared and X-ray spectroscopy to provide estimates of available $\mathrm{P}$ and $\mathrm{K}$. . The spatial variation in the estimates were modelled as mixtures of fixed and random effects by residual maximum likelihood (REML), with the spectroscopic model error accounted for in the parameter estimates. These models where then used to map the $\mathrm{P}$ and $\mathrm{K}$ content by universal kriging together with their kriging variances. Loss functions were computed based on the error distribution and a dose response curve from the literature. We then computed the total loss (compared to perfect information) for each field under VRA of fertilizer and a blanket fertilizer scheme based on the wet chemistry data.

Underestimation of the error variance in four out of eight linear mixed models underlines the importance of error propagation to estimate the short-scale spatial variance of the soil property correctly. As expected, the optimum fertilizer rate when accounting for uncertainty tends towards over-application. The asymmetry of the loss function described that underestimation generally leads to a higher loss compared to overestimation of soil $\mathrm{P}$ and $\mathrm{K}$. The effect of error variance on the expected loss was further found to be dependent on the range of the kriging predictions relative to the parameterization of the dose response curve. There was a financial incentive for VRA of $\mathrm{P}$ fertilizer but not for $\mathrm{K}$. Additionally there is an environmental incentive for VRA of $\mathrm{P}$ 
because much less fertilizer would be applied compared to a blanket fertilizer rate based on wet chemistry data. 